\title{
LINEABILITY OF NON-DIFFERENTIABLE PETTIS PRIMITIVES
}

\author{
B. BONGIORNO, U. B. DARJI, AND L. DI PIAZZA
}

\begin{abstract}
Let $X$ be an infinite-dimensional Banach space. In 1995, settling a long outstanding problem of Pettis, Dilworth and Girardi constructed an $X$-valued Pettis integrable function on $[0,1]$ whose primitive is nowhere weakly differentiable. Using their technique and some new ideas we show that ND, the set of strongly measurable Pettis integrable functions with nowhere weakly differentiable primitives, is lineable, i.e., there is an infinite dimensional vector space whose nonzero vectors belong to ND.
\end{abstract}

\section{INTRODUCTION}

Throughout this note $X$ is an infinite dimensional Banach space. For $X$-valued functions there are essentially two distinct notions of integration: the Bochner integral and the Pettis integral. The latter one includes properly the Bochner integral and preserves some of its good properties, e.g, countable additivity and absolute continuity of the indefinite integral, and convergence theorems. For $X$-valued functions on $[0,1]$ the Bochner indefinite integral is almost everywhere differentiable. As Pettis himself pointed out [17, the same property is not enjoyed by the Pettis integral. An interesting problem left open in [17] was whether the indefinite Pettis integral of a strongly measurable Pettis integrable function $f$ is almost everywhere weakly differentiable (that is, does there exist a set $E \subset[0,1]$ of full measure such that $\int_{0}^{t} x^{*} f$ is differentiable to $x^{*} f$ for each $t \in E$ and for each $x^{*}$ in the dual of $X$ ).

After a string of partial results beginning 1940 ([18, [15], 4, [14]), this problem was settled decisively and beautifully in 1995 by Dilworth and Girardi [5]. They exhibited that every infinite dimensional Banach-space admits a Pettis integrable function from $[0,1]$ into $X$ whose primitive is nowhere weakly differentiable. Their proof is rather flexible and gives the impression that there are many such functions. How does one make such a statement precise? One possibility is to show that in the space of all strongly measurable function from $[0,1]$ into $X$, the set of Pettis integrable functions with nowhere weakly differentiable primitive is a dense $G_{\delta}$ set. This was done by Popa 20 in 2000 . The topology one uses in this setting is the topology generated by the Pettis norm. The shortcoming of this method is that the Pettis norm is not complete [4. Hence, proving that the set of Pettis integrable functions with nowhere weakly differentiable primitive is a dense $G_{\delta}$ set loses some of its significance.

An alternate notion of bigness in Banach space was introduced by Gurarity [11 and followed up in [1] 2] [10. This notion of bigness is of algebraic nature. If $X$ is a Banach space then a subset $M$ of $X$ is lineable if $M \cup\{0\}$ contains an infinite dimensional vector space. If, moreover, this infinite dimensional vector space is closed in the norm topology, then $M$ is said to be spaceable. During the last twenty years many classical, pathological subsets of Banach spaces have been shown to be lineable or spaceable. What is surprising is that most of these sets are far from being vector spaces. For example, Gurarity 12 showed that the space of continuous nowhere differentiable functions on $[0,1]$ is lineable. The spaceability of this set was shown in $[8$. Roderíguez-Piazza 21] showed that every separable Banach space is isometric to a subspace of $C[0,1]$ whose every non-zero element is nowhere differentiable. Later this result was strengthened by Hencl [13] who showed that one can replace "nowhere differentiable" by "nowhere approximately differentiable and nowhere Hölder function". More recently, the spaceability

2010 Mathematics Subject Classification. Primary: 46G10, 28B05 Secondary: 15A03.

Key words and phrases. Pettis Integral, nowhere differentiable, Dvoretzky's theorem, lineable, spaceable.

The second author would like to thank the hospitality of the department of Mathematics of University of Palermo and grant Cori 2013 of the University of Palermo. 
of nowhere integrable functions was shown by Glab, Kaufman and Pellegrini [9]. For a survey of results concerning lineability and spaceability as well as many interesting results and open problems, we refer the reader to the recent paper by Enflo et al. 7 .

In this paper we study, from the viewpoint of lineability, the set of strongly measurable Pettis integrable functions whose Pettis integral is nowhere weakly differentiable. We show (Theorem 2.2) that for every infinite dimensional Banach-space $X$ there is a linearly independent set $\mathcal{V}$ of strongly measurable Pettis integrable $X$-valued functions, satisfying the property that $\mathcal{V}$ has the cardinality of the continuum and for each sequence $\left\{\lambda_{k}\right\} \in \ell_{1}$ and each sequence of $\left\{f_{k}\right\}$ in $\mathcal{V}$, the function $f=\sum_{k=1}^{\infty} \lambda_{k} f_{k}$ is Pettis integrable and its primitive is nowhere weakly differentiable, provided that it is not the zero function. Hence, the lineability of ND follows. Our techniques use ideas of Dilworth and Girardi as well as some interesting applications of the Dvoretzky's theorem and some basic tools of infinite combinatorics. The following problems remain open.

Problem 1.1. Is ND spaceable?

If the answer to the above questions is yes, can more be shown?

Problem 1.2. Let $X$ be an infinite dimensional Banach space. Can every separable Banach space be isometrically embedded into $\mathbf{N D} \cup\{0\}$ ?

The paper is organized as follows: Section 2 contains definitions and some basic facts concerning vector-valued integration. The main result of this article, Theorem 2.2, is also stated there. The rest of the paper concerns the proof of this theorem. We have decided to give proof of the main results in two parts. In Section 3 we give a proof in the case when $X=\ell_{2}$. The reason for giving a separate proof for $\ell_{2}$ is that this case does not use Dvoretzky's Theorem. Hence, the combinatorics and the estimates are easier to follow. Building up on the notation, ideas and techniques of Section 3 , in Section 4 we give the proof of the general case.

\section{BASIC FACTS}

Throughout $X$ is a infinite dimensional Banach space and $X^{*}$ is its dual space. $[0,1]$ is the unit interval of the real line equipped with the Lebesgue measure $\lambda$. By $\mathcal{L}$ we denote the family of all Lebesgue measurable subsets of $[0,1]$.

We recall that a strongly measurable function $f:[0,1] \rightarrow X$ is said to be Bochner integrable in $[0,1]$ if $\int_{0}^{1}\|f\|<\infty$. A function $f:[0,1] \rightarrow X$ is said to be Pettis integrable in $[0,1]$ if $x^{*} f \in L_{1}$ for all $x^{*} \in X^{*}$ and for each $E \in \mathcal{L}$ there exists a vector $x_{E} \in X$ such that $x^{*}\left(x_{E}\right)=\int_{E} x^{*} f$, for all $x^{*} \in X^{*}$. We write $x_{E}=(P) \int_{E} f$ and we call it the Pettis integral of $f$ over $E$. Moreover we call Pettis primitive or simple primitive of $f$ the function $F(t)=(P) \int_{0}^{t} f$. We refer the reader to [3] for basic theory of vector-valued integration.

We use $\mathcal{P}($ resp. $\mathcal{B}$ ) to denote the set of all strongly measurable Pettis integrable (resp. Bochner integrable) functions from $[0,1]$ into $X$. Recall that $\mathcal{B} \subsetneq \mathcal{P}$.

We have the following basic fact.

Lemma 2.1. (see [16, Corollary 5.1]) Let $f:[0,1] \rightarrow X$ be defined by

$$
f=\sum_{k=1}^{\infty} x_{k} \chi_{A_{k}}
$$

where $\left\{A_{k}\right\}$ is a sequence of pairwise disjoint sets of $\mathcal{L}$ and $\left\{x_{n}\right\}$ a sequence in $X$.

(1) $f \in \mathcal{B}$ iff $\sum_{k=1}^{\infty}\left\|x_{k}\right\| \lambda\left(A_{k}\right)<\infty$.

(2) $f \in \mathcal{P}$ is Pettis integrable iff $\sum_{k=1}^{\infty} x_{k} \lambda\left(A_{k}\right)$ is unconditionally convergent in $X$.

Moreover, if either of the integrals exists, then it equals $\sum_{k=1}^{\infty} x_{k} \lambda\left(A_{k}\right)$.

The following is the main theorem of this article.

Theorem 2.2. There exists a set $\mathcal{V} \subset \mathcal{P}$ such that 
(1) $\mathcal{V}$ is the size of the cardinality of the continuum,

(2) $\mathcal{V}$ is linearly independent,

(3) for each sequence $\left\{\lambda_{k}\right\} \in \ell_{1}$ and a sequence of $\left\{f_{k}\right\}$ in $\mathcal{V}$ we have that

$$
f=\sum_{k=1}^{\infty} \lambda_{k} f_{k}
$$

is in $\mathcal{P}$, and

(4) moreover, if $f$ is not the zero function, then the primitive $F$ of $f$, has the property that for all $x \in[0,1]$, we have

$$
\limsup _{h \rightarrow 0}\left\|\frac{F(x+h)-F(x)}{h}\right\|=\infty
$$

\section{The CASE $X=\ell_{2}$}

The proof in the case of $X=\ell_{2}$ is simpler and does not make a use Dvoretzky's theorem. We present this proof first to give the general idea. We will then prove the general case.

We now introduce some terminology and notation necessary for the proof.

By $\{0,1\}^{\infty},\{0,1\}^{<\infty}$ we denote the set of all infinite and the set of all finite (including the empty) sequences of $\{0,1\}$, respectively. For each $\sigma \in\{0,1\}^{<\infty}$, we let $|\sigma|$ denote the length of $\sigma$ and $\sigma i$, $i \in\{0,1\}$, denote the extension of $\sigma$ by $i$. If $\sigma \in\{0,1\}^{<\infty} \cup\{0,1\}^{\infty}$, and $|\sigma| \geq i$, then $\sigma_{\mid i}$ denotes the restriction of $\sigma$ to the first $i$ terms. If $\tau \in\{0,1\}^{<\infty}$, then $[\tau]$ denotes the set of $\sigma \in\{0,1\}^{<\infty}$ which are extension of $\tau$, namely the set of all $\sigma \in\{0,1\}^{<\infty}$ such that $\sigma_{\mid i}=\tau$ where $i=|\tau|$. We let

$$
\mathbf{B}=\left\{(\sigma, i)\left|\sigma \in\{0,1\}^{<\infty}, 0 \leq i \leq\right| \sigma \mid\right\} .
$$

For each $\sigma \in\{0,1\}<\infty$, we define a closed interval $I_{\sigma} \subseteq[0,1]$ of length $2^{-|\sigma|}$ recursively in the following fashion: If $\sigma$ is the empty sequence, then $I_{\sigma}=[0,1]$. In general, if $I_{\sigma}$ is defined then $I_{\sigma 0}$ is the left half of $I_{\sigma}$ and $I_{\sigma 1}$ is the right half of $I_{\sigma}$.

Using the fact that there are nowhere dense sets of positive measure, we obtain a collection $\{A(\sigma, i)$ : $(\sigma, i) \in \mathbf{B}\}$ such that the following conditions hold:

- each $A(\sigma, i)$ is a closed subset of $I_{\sigma}$,

- $\lambda(A(\sigma, i))>0$, and

- if $(\sigma, i) \neq(\tau, j)$, then $A(\sigma, i) \cap A(\tau, j)=\emptyset$.

We also enumerate the standard orthonormal basis of $\ell_{2}$ as $\{e(\sigma, i):(\sigma, i) \in \mathbf{B}\}$ so the following conditions hold:

- each of $e(\sigma, i)$ is of the form $(0, \ldots, 1,0, \ldots)$, and

- $e(\sigma, i) \perp e(\tau, j)$ if $(\sigma, i) \neq(\tau, j)$.

Let $c(\sigma, i) \in \mathbb{R}$ for $(\sigma, i) \in \mathbf{B}$. Then, using these coefficients we define a special type of function as below:

$$
f=\sum_{(\sigma, i) \in \mathbf{B}} c(\sigma, i) \cdot \frac{1}{\lambda(A(\sigma, i))} \cdot e(\sigma, i) \cdot \chi_{A(\sigma, i)} .
$$

Functions of these type will be called basic functions.

If $\tau \in\{0,1\}^{<\infty}$ and $f$ is as above, we define

$$
f_{\mid \tau}=\sum_{(\sigma, i) \in \mathbf{B}, \sigma \in[\tau]} c(\sigma, i) \cdot \frac{1}{\lambda(A(\sigma, i))} \cdot e(\sigma, i) \cdot \chi_{A(\sigma, i)} .
$$

We will freely use the following facts about basic functions. 
Lemma 3.1. Let

$$
f=\sum_{(\sigma, i) \in \mathbf{B}} c(\sigma, i) \cdot \frac{1}{\lambda(A(\sigma, i))} \cdot e(\sigma, i) \cdot \chi_{A(\sigma, i)}
$$

Then,

(1) $f \in \mathcal{P}$ iff $\sum_{(\sigma, i) \in \mathbf{B}} c(\sigma, i)^{2}<\infty$. Moreover, in the case that $f \in \mathcal{P}$, we have that

$$
\left\|\int_{[0,1]} f\right\|=\sqrt{\sum_{(\sigma, i) \in \mathbf{B}} c(\sigma, i)^{2}}
$$

(2) If $I, J$ are subintervals of $[0,1]$ with $I \subseteq J$, then

$$
\left\|\int_{I} f\right\| \leq\left\|\int_{J} f\right\|
$$

(3) If $\tau \in\{0,1\}^{<\infty}$, then

$$
\left\|\int_{I_{\tau}} f\right\| \geq\left\|\int_{I_{\tau}} f_{\mid \tau}\right\|=\sqrt{\sum_{(\sigma, i) \in \mathbf{B}, \sigma \in[\tau]} c(\sigma, i)^{2}} .
$$

Proof. (1) follows from Lemma 2.1 and from the fact that the standard base in $l_{2}$ is unconditional (see [19]). (2) and (3) follow from the fact that

$$
\int_{I} f=\sum_{(\sigma, i) \in \mathbf{B}} c(\sigma, i) \cdot e(\sigma, i) \cdot \frac{\lambda(A(\sigma, i) \cap I)}{\lambda(A(\sigma, i))}
$$

We let

$$
\mathbf{D}=\{\mathbf{n} \mid \mathbf{n}: \mathbb{N} \rightarrow \mathbb{N}, \mathbf{n}(i) \leq i\}
$$

For each $\mathbf{n} \in \mathbf{D}$, we define a special type of basic function. Namely, let $f(\mathbf{n}):[0,1] \rightarrow \ell_{2}$ be defined as

$$
f(\mathbf{n})=\sum_{k=0}^{\infty} \sum_{\sigma \in\{0,1\}^{k}} \frac{1}{(k+1) 2^{k / 2}} \cdot \frac{1}{\lambda(A(\sigma, \mathbf{n}(k)))} \cdot e(\sigma, \mathbf{n}(k)) \cdot \chi_{A(\sigma, \mathbf{n}(k))} .
$$

Lemma 3.2. For each $\mathbf{n} \in \mathbf{D}$, we have that $f(\mathbf{n})$ is Pettis integrable and for $\tau \in\{0,1\}^{i}$, we have that

$$
\left\|\int_{I_{\tau}} f(\mathbf{n})_{\mid \tau}\right\|=2^{-i / 2} \sqrt{\sum_{k=i}^{\infty}\left[\frac{1}{(k+1)^{2}}\right]} .
$$

Lemma 3.3. Let $\left\{\lambda_{i}\right\}$ be a sequence in $\ell_{1}$ and $\left\{f_{i}\right\}$ be such that $f_{i}=f\left(\mathbf{n}_{i}\right)$ for some $\mathbf{n}_{i} \in \mathbf{D}$. Then,

$$
f=\sum_{i=1}^{\infty} \lambda_{i} f_{i} \in \mathcal{P} \quad \text { and } \quad \forall \tau \in\{0,1\}^{<\infty}, \quad\left\|\int_{I_{\tau}} f_{\mid \tau}\right\| \leq \sum_{i=1}^{\infty}\left|\lambda_{i}\right|\left\|\int_{I_{\tau}} f_{i \mid \tau}\right\| .
$$

Proof. We will show that $f$ is Pettis integrable and

$$
\left\|\int_{[0,1]} f\right\| \leq \sum_{i=1}^{\infty}\left|\lambda_{i}\right|\left\|\int_{[0,1]} f_{i}\right\|,
$$

as restricting the function to $I_{\tau}$ does not alter the basic computations. We first note that

$$
f=\sum_{k=0}^{\infty} \sum_{\sigma \in\{0,1\}^{k}} \sum_{j=0}^{k} \frac{1}{(k+1) 2^{k / 2}} \cdot \frac{d(k, j)}{\lambda(A(\sigma, j))} \cdot e(\sigma, j) \cdot \chi_{A(\sigma, j)},
$$


where $d(k, j)=\sum_{\left\{i: \mathbf{n}_{i}(k)=j\right\}} \lambda_{i}$. Hence, $|d(k, j)| \leq \sum_{\left\{i: \mathbf{n}_{i}(k)=j\right\}}\left|\lambda_{i}\right|$. Since $j \neq j^{\prime}$ implies that $\{i$ : $\left.\mathbf{n}_{i}(k)=j\right\} \cap\left\{i: \mathbf{n}_{i}(k)=j^{\prime}\right\}=\emptyset$, we have that

$$
\sum_{j=0}^{k}|d(k, j)|^{2} \leq \sum_{j=0}^{k}\left(\sum_{\left\{i: \mathbf{n}_{i}(k)=j\right\}}\left|\lambda_{i}\right|\right)^{2} \leq\left(\sum_{i=1}^{\infty}\left|\lambda_{i}\right|\right)^{2} .
$$

We note that $f$ is Pettis integrable provided that the series

$$
\sum_{k=0}^{\infty} \sum_{\sigma \in\{0,1\}^{k}} \sum_{j=0}^{k} \frac{|d(k, j)|^{2}}{(k+1)^{2} 2^{k}}<\infty .
$$

Indeed,

$$
\begin{aligned}
\sum_{k=0}^{\infty} \sum_{\sigma \in\{0,1\}^{k}} \sum_{j=0}^{k} \frac{|d(k, j)|^{2}}{(k+1)^{2} 2^{k}} & =\sum_{k=0}^{\infty} \sum_{\sigma \in\{0,1\}^{k}} \frac{1}{(k+1)^{2} 2^{k}} \sum_{j=0}^{k}|d(k, j)|^{2} \\
& \leq \sum_{k=0}^{\infty} \sum_{\sigma \in\{0,1\}^{k}} \frac{1}{(k+1)^{2} 2^{k}}\left(\sum_{i=1}^{\infty}\left|\lambda_{i}\right|\right)^{2} \\
& =\left(\sum_{i=1}^{\infty}\left|\lambda_{i}\right|\right)^{2} \sum_{k=0}^{\infty} \frac{1}{(k+1)^{2}} \\
& <\infty
\end{aligned}
$$

Hence we have that $f$ is Pettis integrable. Moreover, as by Lemma [3.2, $\left\|\int_{[0,1]} f_{i}\right\|=\sqrt{\sum_{k=0}^{\infty} \frac{1}{(k+1)^{2}}}$ for all $i=1,2, \ldots$, we have that

$$
\begin{aligned}
\left\|\int_{[0,1]} f\right\| & =\left(\sum_{k=0}^{\infty} \sum_{\sigma \in\{0,1\}^{k}} \sum_{j=0}^{k} \frac{|d(\sigma, j)|^{2}}{(k+1)^{2} 2^{k}}\right)^{1 / 2} \\
& \leq\left(\left(\sum_{j=1}^{\infty}\left|\lambda_{j}\right|\right)^{2} \sum_{k=0}^{\infty} \frac{1}{(k+1)^{2}}\right)^{1 / 2} \\
& =\sum_{j=1}^{\infty}\left|\lambda_{j}\right| \sqrt{\sum_{k=0}^{\infty} \frac{1}{(k+1)^{2}}} \\
& =\sum_{j=1}^{\infty}\left|\lambda_{j}\right|\left\|\int_{[0,1]} f_{j}\right\|
\end{aligned}
$$

completing the proof.

Proof. (of Theorem 2.2 for $X=\ell_{2}$.) We first obtain a subfamily $\left\{\mathbf{n}_{t}\right\}$ of $\mathbf{D}, 0<t<1$, such that the following condition holds:

$$
s, t \in(0,1) \& s \neq t \Longrightarrow\left\{k: \mathbf{n}_{s}(k)=\mathbf{n}_{t}(k)\right\} \text { is finite. }
$$

This may be done in the following fashion. For each $0<t<1$, consider the line $L_{t}$ going through the origin with slope $t$. For each $k \in \mathbb{N}$ choose $\mathbf{n}_{t}(k) \in \mathbb{N} \cap[0, k]$ so that $L_{t}(k)-1<\mathbf{n}_{t}(k)<L_{t}(k)+1$. Then, the collection $\left\{\mathbf{n}_{t}\right\}$ has the desired property.

Let $\mathcal{V}=\left\{f_{\mathbf{n}_{t}}: t \in(0,1)\right\}$. We will now show that $\mathcal{V}$ has the desired properties. 
It is clear that $\mathcal{V}$ has the cardinality that of the continuum. By (3.1) it follows that no non-trivial finite linear combination of elements of $\left\{f_{\mathbf{n}_{t}}\right\}$ is the zero function. Hence, $\mathcal{V}$ is linearly independent. That $\mathcal{V}$ satisfies conclusion (3) of the theorem follows from Lemma 3.3 .

Finally, to verify conclusion (4) of the theorem, let us show that if $f$ is not the zero function. then for all $x \in[0,1]$, we have

$$
\lim _{h \rightarrow 0}\left\|\frac{F(x+h)-F(x)}{h}\right\|=\infty,
$$

where $F$ is the primitive of $f$. As $f$ is not the zero function, we may assume, by renumeration, that all of the $\left\{f_{i}\right\}$ 's are distinct and $\lambda_{1} \neq 0$. Moreover, we lose no generality by assuming that $\lambda_{1}=1$. Let $i_{0}$ be such that $\sum_{i=i_{0}}^{\infty}\left|\lambda_{i}\right|<\frac{1}{2}$. Let $t_{i}$ be such that $f_{i}=f_{\mathbf{n}_{t_{i}}}$. Let $M>0$. By our choice of $\left\{\mathbf{n}_{t_{i}}\right\}$, we have that there exists a positive integer $l$ such that for all $k \geq l, \sigma \in\{0,1\}^{k}$ and $1 \leq i<i^{\prime} \leq i_{0}$, we have that $\mathbf{n}_{i}(k) \neq \mathbf{n}_{i^{\prime}}(k)$ and hence $e\left(\sigma, n_{i}(k)\right) \perp e\left(\sigma, n_{i^{\prime}}(k)\right)$. Choose $k_{0}$ so large so that $k_{0}>l$ and $2^{i / 2-3} \sqrt{\sum_{k=i}^{\infty} \frac{1}{(k+1)^{2}}}>M$ for all $i>k_{0}$. Let $\delta=2^{-k_{0}}$. Let $h \in \mathbb{R}$ be such that $0<|h|<\delta$. We wish to show that

$$
\left\|\frac{F(x+h)-F(x)}{h}\right\|>M .
$$

Without loss of generality, we may assume that $h>0$. Let $j$ be the smallest positive integer so that there is $\tau \in\{0,1\}^{j}$ so that $I_{\tau} \subseteq[x, x+h]$. We note that $j>k_{0}$ and $h<4 \cdot 2^{-j}$.

$$
\begin{aligned}
\|F(x+h)-F(x)\| & =\left\|\int_{[x, x+h]} f\right\| \\
& \geq\left\|\int_{I_{\tau}} f\right\| \\
& \geq\left\|\int_{I_{\tau}} f_{\mid \tau}\right\| \\
& =\left\|\int_{I_{\tau}} \sum_{i=1}^{i_{0}} \lambda_{i} f_{i \mid \tau}+\int_{I_{\tau}} \sum_{i=i_{0}}^{\infty} \lambda_{i} f_{i \mid \tau}\right\| \\
& \geq\left\|\int_{I_{\tau}} \sum_{i=1}^{i_{0}} \lambda_{i} f_{i \mid \tau}\right\|-\left\|\int_{I_{\tau}} \sum_{i=i_{0}}^{\infty} \lambda_{i} f_{i \mid \tau}\right\| \\
& \geq\left\|\int_{I_{\tau}} \sum_{i=1}^{i_{0}} \lambda_{i} f_{i \mid \tau}\right\|-\sum_{i=i_{0}}^{\infty}\left|\lambda_{i}\right|\left\|f_{I_{\tau}} f_{i \mid \tau}\right\| \\
& \geq\left\|\int_{I_{\tau}} f_{1 \mid \tau}\right\|-\sum_{i=i_{0}}^{\infty}\left|\lambda_{i}\right|\left\|\int_{I_{\tau}} f_{i \mid \tau}\right\| \\
& =\left(1-\sum_{i=i_{0}}^{\infty}\left|\lambda_{i}\right|\right)^{-j / 2} \sqrt{\sum_{k=j}^{\infty}\left[\frac{1}{(k+1)^{2}}\right]}
\end{aligned}
$$

Let us give some explanations for the above inequalities. Let us first observe that $f$ is a basic function. Inequalities (3.2) and (3.3) above follow from Lemma 3.1. Inequality (3.4) is simply the triangle inequality. Meanwhile, inequality (3.5) follows from Lemma 3.3. Inequality (3.6) holds because $j>k_{0}>l$ which implies that for all $1 \leq i<i^{\prime} \leq i_{0}$ we have that $e\left(\sigma, n_{i}(|\sigma|)\right) \perp e\left(\sigma, n_{i^{\prime}}(|\sigma|)\right)$ for all $\sigma$ extensions of $\tau$ and $(\sigma, k) \in \mathbf{B}$. Finally, estimate (3.7) follows from Lemma 3.2 Now using the above 
estimate and the fact that $h<4 \cdot 2^{-j}$, we obtain that

$$
\begin{aligned}
\left\|\frac{F(x+h)-F(x)}{h}\right\| & >\frac{\left(1-\sum_{i=i_{0}}^{\infty}\left|\lambda_{i}\right|\right) 2^{-j / 2} \sqrt{\sum_{k=j}^{\infty}\left[\frac{1}{(k+1)^{2}}\right]}}{4 \cdot 2^{-j}} \\
& \geq 2^{j / 2-3} \sqrt{\sum_{k=j}^{\infty} \frac{1}{(k+1)^{2}}} \\
& >M .
\end{aligned}
$$

\section{The General CASE}

The proof of the general case is similar to the $\ell_{2}$ case, the main difference being the lack of orthonormal basis in an arbitrary Banach space $X$. We use the notation and the terminology of the previous section with one exception: $\{e(\sigma, i):(\sigma, i) \in \mathbf{B}\}$ will have to be constructed with the help of Dvoretzky's theorem. The calculations are also more involved.

Lemma 4.1. Let $\left\{b_{n}\right\}$ be a basic sequence in $X$. Then there is $K>1$ such that for all $\lambda_{0}, \lambda_{1} \ldots \in \mathbb{R}$ for which $\sum_{i=0}^{\infty} \lambda_{i} b_{i}$ converges in $X$ we have that

$$
\left\|\sum_{i=0}^{\infty} \lambda_{i} b_{i}\right\| \geq \frac{1}{K}\left\|\sum_{i=k}^{l} \lambda_{i} b_{i}\right\| \text { for all } k<l \in \mathbb{N} \cup\{\infty\} .
$$

Proof. Let $Y$ be the subspace of $X$ generated $\left\{b_{n}\right\}$. Let $\pi_{k}: Y \rightarrow Y$ be the natural projection defined as

$$
\pi_{k}\left(\sum_{i=0}^{\infty} \lambda_{i} b_{i}\right)=\sum_{i=0}^{k} \lambda_{i} b_{i}
$$

( $\pi_{\infty}$ is simply the identity map.) It is well-known that $\pi_{k}: Y \rightarrow Y$ is a bounded linear map. Moreover, it is known that $\left\{\pi_{k}: k \in \mathbb{N} \cup\{\infty\}\right\}$ is uniformly bounded. For each $k<l \in \mathbb{N} \cup\{\infty\}$, let

$$
q_{k, l}\left(\sum_{i=0}^{\infty} \lambda_{i} b_{i}\right)=\sum_{i=k}^{l} \lambda_{i} b_{i} .
$$

Then, $q_{k, l}=\pi_{l}-\pi_{k-1}$. Hence, the family $\left\{q_{k, l}\right\}$ is uniformly bounded and the lemma follows.

Throughout this section $\left\{b_{n}\right\}$ and $K$ are as above in the Lemma 4.1 ,

We now proceed to construct $\{e(\sigma, j):(\sigma, i) \in \mathbf{B}\}$. Let $\left\{n_{k}\right\}_{k=0}^{\infty}$ be a strictly increasing sequence such that $n_{0}=0$. Let

$$
\mathbf{B}_{k}=\left\{(\sigma, i): \sigma \in\{0,1\}^{\left[n_{k}, n_{k+1}\right)}, \quad 0 \leq i \leq|\sigma|\right\} .
$$

We call $\mathbf{B}_{k}$ the $k^{\text {th }}$ block.

The following lemma follows from Dvoretzky's theorem (see [6]).

Lemma 4.2. Let $\left\{n_{k}\right\}_{k=0}^{\infty},\left\{m_{k}\right\}_{k=0}^{\infty}$ and $B_{k}$ be as above. There exits $\left\{e(\sigma, i):(\sigma, i) \in B_{k}\right\}$ such that the following conditions hold:

$$
\begin{gathered}
\left\{e(\sigma, i):(\sigma, i) \in \mathbf{B}_{k}\right\} \subseteq \operatorname{span}\left\{b_{j}: j \in\left[m_{k}, m_{k+1}\right)\right\} \\
\forall \lambda(\sigma, i) \in \mathbb{R} \quad \sqrt{\sum_{(\sigma, i) \in \mathbf{B}_{k}} \lambda(\sigma, i)^{2}} \geq\left\|\sum_{(\sigma, i) \in \mathbf{B}_{k}} \lambda(\sigma, i) e(\sigma, i)\right\| \geq \frac{1}{2} \sqrt{\sum_{(\sigma, i) \in \mathbf{B}_{k}} \lambda(\sigma, i)^{2}} .
\end{gathered}
$$


As before, we let

$$
f=\sum_{(\sigma, i) \in \mathbf{B}} c(\sigma, i) \cdot \frac{1}{\lambda(A(\sigma, i))} \cdot e(\sigma, i) \cdot \chi_{A(\sigma, i)}
$$

and for $\tau \in\{0,1\}^{n_{k}}$, let

$$
f_{\mid \tau}=\sum_{(\sigma, i) \in \mathbf{B}, \sigma \in[\tau]} c(\sigma, j) \cdot \frac{1}{\lambda(A(\sigma, j))} \cdot e(\sigma, j) \cdot \chi_{A(\sigma, j)} .
$$

Lemma 4.3. Let $f$ and $f_{\mid \tau}$ be as above with $\tau \in\{0,1\}^{n_{k}}$.

(1) If $\sum_{j=0}^{\infty} \sqrt{\sum_{(\sigma, i) \in \mathbf{B}_{j}} c(\sigma, i)^{2}}<\infty$, then $f \in \mathcal{P}$.

$$
\frac{1}{2 K} \sqrt{\sum_{(\sigma, i) \in \mathbf{B}_{k}, \sigma \in[\tau]} c(\sigma, i)^{2}} \leq\left\|\int_{I_{\tau}} f_{\mid \tau}\right\| \leq \sum_{j=k}^{\infty} \sqrt{\sum_{(\sigma, i) \in \mathbf{B}_{j}, \sigma \in[\tau]} c(\sigma, i)^{2}}
$$

$$
\left\|\int_{I_{\tau}} f\right\| \geq \frac{1}{K}\left\|\int_{I_{\tau}} f_{\mid \tau}\right\|
$$

Proof. To see (1) recall that $f \in \mathcal{P}$ provided that $\sum_{(\sigma, i) \in \mathbf{B}} c(\sigma, i) e(\sigma, i)$ converges unconditionally. To see this, let $\epsilon(\sigma, i) \in\{-1,1\}$ for all $(\sigma, i) \in \mathbf{B}$. Then,

$$
\begin{aligned}
\left\|\sum_{(\sigma, i) \in \mathbf{B}} \epsilon(\sigma, i) c(\sigma, i) e(\sigma, i)\right\| & \leq \sum_{j=0}^{\infty}\left\|\sum_{(\sigma, i) \in \mathbf{B}_{j}} \epsilon(\sigma, i) c(\sigma, i) e(\sigma, i)\right\| \\
& \leq \sum_{j=0}^{\infty} \sqrt{\sum_{(\sigma, i) \in \mathbf{B}_{j}} c(\sigma, i)^{2}} \\
& <\infty .
\end{aligned}
$$

Note that inequality (4.1) is simply the triangle inequality and inequality (4.2) follows from Lemma 4.2 . Let us now verify (2). To obtain the lower bound, we observe that

$$
\begin{aligned}
\left\|\int_{I_{\tau}} f_{\mid \tau}\right\| & =\left\|\sum_{(\sigma, i) \in \mathbf{B}, \sigma \in[\tau]} c(\sigma, i) \cdot \frac{\lambda\left(A(\sigma, i) \cap I_{\tau}\right)}{\lambda(A(\sigma, i))} \cdot e(\sigma, i)\right\| \\
& =\left\|\sum_{(\sigma, i) \in \mathbf{B}, \sigma \in[\tau]} c(\sigma, i) \cdot e(\sigma, i)\right\| \\
& =\left\|\sum_{j=k}^{\infty} \sum_{(\sigma, i) \in \mathbf{B}_{j}, \sigma \in[\tau]} c(\sigma, i) \cdot e(\sigma, i)\right\| \\
& \geq \frac{1}{K}\left\|\sum_{(\sigma, i) \in \mathbf{B}_{k}, \sigma \in[\tau]} c(\sigma, i) \cdot e(\sigma, i)\right\| \\
& \geq \frac{1}{2 K} \sqrt{\sum_{(\sigma, i) \in \mathbf{B}_{k}, \sigma \in[\tau]} c(\sigma, i)^{2}}
\end{aligned}
$$


Inequality (4.7) follows from the fashion in which $e(\sigma, i)$ 's were chosen and Lemma 4.1, Meanwhile Inequality (4.8) follows from Lemma 4.2. To obtain the upper bound, we observe that

$$
\begin{aligned}
\left\|\int_{I_{\tau}} f_{\mid \tau}\right\| & =\left\|\sum_{j=k}^{\infty} \sum_{(\sigma, i) \in \mathbf{B}_{j}, \sigma \in[\tau]} c(\sigma, i) \cdot e(\sigma, i)\right\| \\
& \leq \sum_{j=k}^{\infty}\left\|\sum_{(\sigma, i) \in \mathbf{B}_{j}, \sigma \in[\tau]} c(\sigma, i) \cdot e(\sigma, i)\right\| \\
& \leq \sum_{j=k}^{\infty} \sqrt{\sum_{(\sigma, i) \in \mathbf{B}_{j}, \sigma \in[\tau]} c(\sigma, i)^{2}} .
\end{aligned}
$$

Inequality (4.10) simply is the triangle inequality. Inequality (4.11) follows from Lemma 4.2 .

Finally, let us now prove (3). We note that

$$
\begin{aligned}
\left\|\int_{I_{\tau}} f\right\| & =\left\|\sum_{(\sigma, i) \in \mathbf{B}, \tau \in[\sigma]} c(\sigma, i) \cdot \frac{\lambda\left(A(\sigma, i) \cap I_{\tau}\right)}{\lambda(A(\sigma, i))} \cdot e(\sigma, i)+\sum_{(\sigma, i) \in \mathbf{B}, \sigma \in[\tau]} c(\sigma, i) \cdot e(\sigma, i)\right\| \\
& =\left\|\sum_{j=0}^{k-1} \sum_{(\sigma, i) \in \mathbf{B}_{j}, \tau \in[\sigma]} c(\sigma, i) \cdot \frac{\lambda\left(A(\sigma, i) \cap I_{\tau}\right)}{\lambda(A(\sigma, i))} \cdot e(\sigma, i)+\sum_{j=k}^{\infty} \sum_{(\sigma, i) \in \mathbf{B}_{j}, \sigma \in[\tau]} c(\sigma, i) \cdot e(\sigma, i)\right\| \\
& \geq \frac{1}{K}\left\|\sum_{j=k}^{\infty} \sum_{(\sigma, i) \in \mathbf{B}_{j}, \sigma \in[\tau]} c(\sigma, i) \cdot e(\sigma, i)\right\| \\
& =\frac{1}{K}\left\|\int_{I_{\tau}} f_{\mid \tau}\right\|
\end{aligned}
$$

Again in the inequalities above we make a use of the fashion in which $e(\sigma, i)$ 's where chosen and Lemma 4.1 .

Recall that

$$
\mathbf{D}=\{\mathbf{n} \mid \mathbf{n}: \mathbb{N} \rightarrow \mathbb{N}, \mathbf{n}(i) \leq i\}
$$

and

$$
f(\mathbf{n})=\sum_{k=0}^{\infty} \sum_{\sigma \in\{0,1\}^{k}} \frac{1}{(k+1) 2^{k / 2}} \cdot \frac{1}{\lambda(A(\sigma, \mathbf{n}(k)))} \cdot e(\sigma, \mathbf{n}(k)) \cdot \chi_{A(\sigma, \mathbf{n}(k))} .
$$

For the sake of notational convenience, for each $k \in \mathbb{N}$, let

$$
u_{k} \equiv \sqrt{\sum_{i=n_{k}}^{n_{k+1}-1} \frac{1}{(i+1)^{2}}} .
$$

Henceforth, we assume that $\left\{n_{k}\right\}$ is rapidly increasing so that

$$
u_{k+1}<\frac{1}{3} u_{k} .
$$

Lemma 4.4. For each $\mathbf{n} \in \mathbf{D}$, we have that $f(\mathbf{n})$ is Pettis integrable and for $\tau \in\{0,1\}^{n_{k}}$, we have that

$$
\frac{1}{2 K} 2^{-\frac{n_{k}}{2}} u_{k} \leq\left\|\int_{I_{\tau}} f(\mathbf{n})_{\mid \tau}\right\| \leq \frac{3}{2} 2^{-\frac{n_{k}}{2}} u_{k}
$$


Proof. Let us compute the lower estimate first. By Lemma 4.3 we have that

$$
\begin{aligned}
\left\|\int_{I_{\tau}} f(\mathbf{n})_{\mid \tau}\right\| & \geq \frac{1}{2 K} \sqrt{\sum_{i=n_{k}}^{n_{k+1}-1} 2^{i-n_{k}}\left[\frac{1}{(i+1) 2^{i / 2}}\right]^{2}} \\
& =\frac{1}{2 K} 2^{-\frac{n_{k}}{2}} \sqrt{\sum_{i=n_{k}}^{n_{k+1}-1}\left[\frac{1}{(i+1)}\right]^{2}} \\
& =\frac{1}{2 K} 2^{-\frac{n_{k}}{2}} u_{k} .
\end{aligned}
$$

Again, using the upper estimate in Lemma 4.3 we have that

$$
\begin{aligned}
\left\|\int_{I_{\tau}} f(\mathbf{n})_{\mid \tau}\right\| & \leq \sum_{j=k}^{\infty} \sqrt{\sum_{i=n_{k}}^{n_{k+1}-1} 2^{i-n_{k}}\left[\frac{1}{(i+1) 2^{i / 2}}\right]^{2}} \\
& \leq 2^{-n_{k} / 2} \sum_{j=k}^{\infty} \sqrt{\sum_{i=n_{k}}^{n_{k+1}-1}\left[\frac{1}{(i+1)^{2}}\right]^{2}} \\
& \leq 2^{-n_{k} / 2} \sum_{j=k}^{\infty} u_{j} \\
& \leq 2^{-n_{k} / 2} \sum_{j=k}^{\infty} u_{k}\left(\frac{1}{3}\right)^{j-k} \\
& \leq \frac{3}{2} 2^{-n_{k} / 2} u_{k} .
\end{aligned}
$$

Lemma 4.5. Let $\left\{\lambda_{i}\right\}$ be a sequence in $\ell_{1}$ and $\left\{f_{i}\right\}$ be such that $f_{i}=f\left(\mathbf{n}_{i}\right)$ for some $\mathbf{n}_{i} \in \mathbf{D}$. Then,

$$
f=\sum_{i=1}^{\infty} \lambda_{i} f_{i} \in \mathcal{P} \quad \text { and } \quad \forall \tau \in\{0,1\}^{<\infty}, \quad\left\|\int_{I_{\tau}} f\right\| \leq \sum_{i=1}^{\infty}\left|\lambda_{i}\right|\left\|\int_{I_{\tau}} f_{i}\right\| .
$$

Proof. We only need to show that $f \in \mathcal{P}$ as the second part simply follows from the general theory of integration. We first note that

$$
f=\sum_{k=0}^{\infty} \sum_{\sigma \in\{0,1\}^{k}} \sum_{j=0}^{k} \frac{1}{(k+1) 2^{k / 2}} \cdot \frac{d(k, j)}{\lambda(A(\sigma, j))} \cdot e(\sigma, j) \cdot \chi_{A(\sigma, j)},
$$

where $d(k, j)=\sum_{\left\{i: \mathbf{n}_{i}(k)=j\right\}} \lambda_{i}$. Hence, $|d(k, j)| \leq \sum_{\left\{i: \mathbf{n}_{i}(k)=j\right\}}\left|\lambda_{i}\right|$. Since $j \neq j^{\prime}$ implies that $\{i$ : $\left.\mathbf{n}_{i}(k)=j\right\} \cap\left\{i: \mathbf{n}_{i}(k)=j^{\prime}\right\}=\emptyset$, we have that

$$
\sum_{j=0}^{k}|d(k, j)|^{2} \leq \sum_{j=0}^{k}\left(\sum_{\left\{i: \mathbf{n}_{i}(k)=j\right\}}\left|\lambda_{i}\right|\right)^{2} \leq\left(\sum_{j=0}^{\infty}\left|\lambda_{j}\right|\right)^{2} .
$$

Let us rewrite $f$. For $(\sigma, j) \in \mathbf{B}$, let $c(\sigma, j)=\frac{1}{(|\sigma|+1) 2^{|\sigma| / 2}} \cdot d(|\sigma|, j)$. Then

$$
f=\sum_{(\sigma, j) \in \mathbf{B}} c(\sigma, j) \cdot \frac{1}{\lambda(A(\sigma, j))} \cdot e(\sigma, j) \cdot \chi_{A(\sigma, j)} .
$$


By (1) of Lemma 4.3, we have that $f$ is Pettis integrable provided that $\sum_{k=0}^{\infty} \sqrt{\sum_{(\sigma, j) \in \mathbf{B}_{k}} c(\sigma, j)^{2}}<\infty$. Indeed,

$$
\begin{aligned}
\sum_{k=0}^{\infty} \sqrt{\sum_{(\sigma, j) \in \mathbf{B}_{k}} c(\sigma, j)^{2}} & =\sum_{k=0}^{\infty} \sqrt{\sum_{i=n_{k}}^{n_{k+1}-1} \sum_{\sigma \in\{0,1\}^{i}} \sum_{j=0}^{i} \frac{1}{2^{i}(i+1)^{2}} d(i, j)^{2}} \\
& \leq \sum_{k=0}^{\infty} \sqrt{\sum_{i=n_{k}}^{n_{k+1}-1} \sum_{\sigma \in\{0,1\}^{i}} \frac{1}{2^{i}(i+1)^{2}}\left(\sum_{j=1}^{\infty}\left|\lambda_{j}\right|\right)^{2}} \\
& =\sum_{k=0}^{\infty} \sqrt{\sum_{i=n_{k}}^{n_{k+1}-1} \frac{1}{(i+1)^{2}}\left(\sum_{j=1}^{\infty}\left|\lambda_{j}\right|\right)^{2}} \\
& =\left(\sum_{j=1}^{\infty}\left|\lambda_{j}\right|\right) \sum_{k=0}^{\infty} \sqrt{\sum_{i=n_{k}}^{n_{k+1}-1} \frac{1}{(i+1)^{2}}} \\
& =\sum_{k=0}^{\infty} u_{k} \\
& <\frac{3}{2} u_{0}<\infty .
\end{aligned}
$$

Proof. (of Theorem 2.2 for $X$ arbitrary infinite dimensional Banach space.)

As before let $\left\{\mathbf{n}_{t}\right\}, 0<t<1$, be a subfamily of $\mathbf{D}$ that satisfies the following condition:

$$
s, t \in(0,1) \& s \neq t \Longrightarrow\left\{k: \mathbf{n}_{s}(k)=\mathbf{n}_{t}(k)\right\} \text { is finite. }
$$

Let $\mathcal{V}=\left\{f_{\mathbf{n}_{t}}: t \in(0,1)\right\}$. We will now show that $\mathcal{V}$ has the desired properties.

It is clear that $\mathcal{V}$ has the cardinality that of the continuum. As before we have that $\mathcal{V}$ is linearly independent. That $\mathcal{V}$ satisfies conclusion (3) of the theorem follows from Lemma 4.5.

Finally, to verify conclusion (4) of the theorem, let us show that if $f$ is not the zero function, then for all $x \in[0,1]$, we have

$$
\limsup _{h \rightarrow 0}\left\|\frac{F(x+h)-F(x)}{h}\right\|=\infty
$$

where $F$ is the primitive of $f$. As $f$ is not the zero function, we may assume, by renumeration, that all of the $\left\{f_{i}\right\}$ 's are distinct and $\lambda_{1} \neq 0$. Moreover, we lose no generality by assuming that $\lambda_{1}=1$. Let $i_{0}$ be such that $\sum_{i=i_{0}}^{\infty}\left|\lambda_{i}\right|<\frac{1}{8 K}$. Let $t_{i}$ be such that $f_{i}=f_{\mathbf{n}_{t_{i}}}$. Let $M>0$. By our choice of $\left\{\mathbf{n}_{t_{i}}\right\}$, we have that there exists a positive integer $l$ such that for all $k \geq l, \sigma \in\{0,1\}^{k}$ and $1 \leq i<i^{\prime} \leq i_{0}$, we have that $\mathbf{n}_{t_{i}}(\sigma) \neq \mathbf{n}_{t_{i^{\prime}}}(\sigma)$. Choose $k$ so large so that $k>l$ and $\frac{1}{4 K^{2}} 2^{\frac{n_{k}}{2}} u_{k}>M$. Let $\tau \in\{0,1\}^{n_{k}}$ be such that $x \in I_{\tau}$. We wish to show that

$$
\frac{\left\|\int_{I_{\tau}} f\right\|}{\left|I_{\tau}\right|}>M .
$$

By (3) of Lemma 4.3, we have that $\left\|\int_{I_{\tau}} f\right\| \geq \frac{1}{K}\left\|\int_{I_{\tau}} f_{\mid \tau}\right\|$. Hence, it suffices to show that

$$
\frac{\left\|\int_{I_{\tau}} f_{\mid \tau}\right\|}{\left|I_{\tau}\right|}>K M .
$$


We let

$$
\left\|\int_{I_{\tau}} f_{\mid \tau}\right\|=\left\|\int_{I_{\tau}}\left(\sum_{i=1}^{\infty} \lambda_{i} f_{i}\right)_{\mid \tau}\right\| \geq L_{1}-L_{2}
$$

where

$$
L_{1}=\left\|\int_{I_{\tau}}\left(\sum_{i=1}^{i_{0}} \lambda_{i} f_{i}\right)_{\mid \tau}\right\|, \quad L_{2}=\left\|\int_{I_{\tau}}\left(\sum_{i=i_{0}+1}^{\infty} \lambda_{i} f_{i}\right)_{\mid \tau}\right\| .
$$

Let us write

$$
\begin{aligned}
\sum_{i=1}^{i_{0}} \lambda_{i} f_{i} & =\sum_{(\sigma, j) \in \mathbf{B}} c(\sigma, j) \cdot d(\sigma, j) \cdot \frac{1}{\lambda(A(\sigma, j))} \cdot e(\sigma, j) \cdot \chi_{A(\sigma, j)} \text { where } \\
c(\sigma, j) & =c(\sigma)=\frac{1}{(|\sigma|+1) 2^{\frac{|\sigma|}{2}}}, \text { and } d(\sigma, j)=\sum_{\left\{1 \leq i \leq i_{0}: \mathbf{n}_{i}(|\sigma|)=j\right\}} \lambda_{i}
\end{aligned}
$$

if $\left\{1 \leq i \leq i_{0}: \mathbf{n}_{i}(|\sigma|)=j\right\}$ is not empty, $d(\sigma, j)=0$ otherwise.

Then, we have that

$$
\begin{aligned}
L_{1} & =\left\|\int_{I_{\tau}}\left(\sum_{i=1}^{i_{0}} \lambda_{i} f_{i}\right)_{\mid \tau}\right\| \\
& =\left\|\int_{I_{\tau}}\left(\sum_{(\sigma, j) \in \mathbf{B}} c(\sigma, j) \cdot d(\sigma, j) \cdot \frac{1}{\lambda(A(\sigma, j))} \cdot e(\sigma, j) \cdot \chi_{A(\sigma, j)}\right)_{\mid \tau}\right\| \\
& \geq \frac{1}{2 K} \sqrt{\sum_{(\sigma, j) \in \mathbf{B}_{k}, \sigma \in[\tau]} c(\sigma, j)^{2} \cdot d(\sigma, j)^{2}} \\
& \geq \frac{1}{2 K} \sqrt{\sum_{i=n_{k}}^{n_{k+1}-1} \sum_{\sigma \in\{0,1\}^{i}, \sigma \in[\tau]} c(\sigma)^{2}} \\
& =\frac{1}{2 K} \sqrt{\sum_{i=n_{k}}^{n_{k+1}-1} \sum_{\sigma \in\{0,1\}^{i}, \sigma \in[\tau]}\left(\frac{1}{(|\sigma|+1) 2^{\frac{|\sigma|}{2}}}\right)^{2}} \\
& =\frac{1}{2 K} \sqrt{\sum_{i=n_{k}}^{n_{k+1}-1} 2^{i-n_{k}}\left[\frac{1}{(i+1) 2^{i / 2}}\right]^{2}}=\frac{2^{-\frac{n_{k}}{2}}}{2 K} u_{k} .
\end{aligned}
$$

Let us give some justifications for the inequalities. Inequality (4.12) follows from (2) of Lemma 4.3 , Let us now explain why inequality (4.13) holds. We note that if $1 \leq i<i^{\prime} \leq i_{0}$ and $\sigma \in[\tau]$, then $n_{t_{i}}(|\sigma|) \neq n_{t_{i^{\prime}}}(|\sigma|)$. Hence, $\sigma \in[\tau]$ implies that $d(\sigma, j)=0$ or $\lambda_{i}$ for some $1 \leq i \leq i_{0}$. Moreover, for every $\sigma \in[\tau]$ there is a $j$ so that $d(\sigma, j)=\lambda_{1}=1$. Hence inequality (4.13) holds. The rest of the equalities are basic computations. 
We next obtain an upper estimate of $L_{2}$.

$$
\begin{aligned}
L_{2} & =\left\|\int_{I_{\tau}}\left(\sum_{i=i_{0}+1}^{\infty} \lambda_{i} f_{i}\right)_{\mid \tau}\right\| \\
& =\left\|\int_{I_{\tau}} \sum_{i=i_{0}+1}^{\infty} \lambda_{i}\left(f_{i}\right)_{\mid \tau}\right\| \\
& \leq \sum_{i=i_{0}+1}^{\infty}\left|\lambda_{i}\right|\left\|\int_{I_{\tau}}\left(f_{i}\right)_{\mid \tau}\right\| \\
& \leq\left(\sum_{i=i_{0}+1}^{\infty}\left|\lambda_{i}\right|\right)\left(\frac{3}{2} 2^{-\frac{n_{k}}{2}} u_{k}\right) \\
& \leq \frac{1}{8 K} \frac{3}{2} 2^{-\frac{n_{k}}{2}} u_{k}<\frac{1}{4 K} 2^{-\frac{n_{k}}{2}} u_{k}
\end{aligned}
$$

We note that inequality (4.17) follows from Lemma 4.5 and inequality (4.18) follows from Lemma 4.4,

Putting these estimates together we get that

$$
\left\|\int_{I_{\tau}} f_{\mid \tau}\right\| \geq L_{1}-L_{2}>\frac{1}{4 K} 2^{-\frac{n_{k}}{2}} u_{k}
$$

This, in turn, implies that

$$
\frac{\left\|\int_{I_{\tau}} f_{\mid \tau}\right\|}{\left|I_{\tau}\right|}>\frac{\frac{1}{4 K} 2^{-\frac{n_{k}}{2}} u_{k}}{2^{-n_{k}}} \geq \frac{1}{4 K} 2^{\frac{n_{k}}{2}} u_{k}>K M,
$$

completing the proof.

\section{REFERENCES}

[1] R. M. Aron, V. I. Gurariy, J. B. Seoane-Sepúlveda, Lineability and spaceability of sets of functions on $\mathbb{R}$. Proc. Amer. Math. Soc. 133 (2005), 795-803.

[2] R. M. Aron, D. Pérez-Garcá, J. B. Seoane-Sepúlveda, Algebrability of the set of non- convergent Fourier series. Studia Math. 175 (2006) no 1, 83-90.

[3] J. Diestel, J. J. Uhl, Vector measures, Math. Surveys, 15 (1977).

[4] S. J. Dilworth, M. Girardi, Bochner vs. Pettis norm: examples and results. Banach spaces (Mrida, 1992), Contemp. Math., 144, Amer. Math. Soc., Providence, RI, 1993, 69-80.

[5] S. J. Dilworth, M. Girardi, Nowhere weak differentiability of the Pettis integral, Quaest. Math., 18 (1995), 365-380.

[6] A. Dvoretzky, Some results on convex bodies and Banach spaces, Proc. Internat. Sympos. Linear Spaces (Jerusalem, 1960), Jerusalem Academic Press, (1961), 123-160.

[7] P. H. Enflo, V. I. Gurariy, J. B. Seoane-Sepúlveda, Some results and open questions on spaceability in function space. Trans. Amer. Math. Soc. 366 no 2 (2014), 611-625.

[8] V. Fonf, V. I. Gurariy, V. Kadeč. An infinite dimensional subspace of $C[0,1]$ consisting of nowhere differentiable functions. C. R. Acad. Bulgare Sci. 52 (1999) no 11-12, 13-16.

[9] S. Głąb, P. L. Kaufmann, L. Pellegrini, Spaceability and algebrability of sets of nowhere integrable functions. Proc. Amer. Math. Soc. 141 (2013), no. 6, 2025-2037.

[10] V. I. Gurariy, L. Quarta. On lineability of sets of continuous functions. J. Math. Anal. Appl. 294 (2004), 62-72.

[11] V. I. Gurariy, Subspaces and bases in spaces of continuous functions. (Russian). Dokl. Akad. Nauk SSSR 167 (1966), 971-973.

[12] V. I. Gurariy, Linear spaces composed of nondifferentiable functions. C. R. Acad. Bulgare Sci. 44, (1991) no 5, 13-16.

[13] S. Hencl, Isometrical embeddings of separable Banach spaces into the set of nowhere approximately differentiable and nowhere H ölder functions. Proc. Amer. Math. Soc. 128 (2000), no. 12, 3505-3511.

[14] V. M. Kadets, Non-differentiable indefinite Pettis integral, Quaest. Math. 17 (1994), no. 2 (1994), no. 2, 137-139.

[15] M. E. Munroe, A note on weak differentiability of Pettis integrals, Bull. Amer. Math. Soc. 52 (1946), 167-175.

[16] K. Musial, Topics in the theory of Pettis integration, Rend. Istit. Mat. Univ. Trieste 23 (1991), 177-262.

[17] B. J. Pettis, On integration in vector spaces, Trans. Amer. Math. Soc. 44 (1938), 227-304.

[18] R. S. Phillips, Integration in a convex linear topological space, Trans. Amer. Math. Soc. 47 (1940), 114-145.

[19] I. Singer, Bases in Banach spaces I, Springer-Verlag, Berlin and New York (1981). 
[20] D. Popa, Sets which are dense in the space of all Pettis integrable functions, Quaest. Math. 23 (2000), no. 4, 525-28.

[21] L. Rodríguez-Piazza, Every separable Banach space is isometric to a space of continuous nowhere differentiable functions. Proc. Amer. Math. Soc. 123 (1995), no. 12, 3649-3654.

Department of Mathematics, University of Palermo, Via Archirafi 34, 90123 Palermo, Italy

E-mail address: bbongi@math.unipa.it

Department of Mathematics, University of Louisville, Louisville, KY 40292, USA

E-mail address: ubdarj01@louisville.edu

Department of Mathematics, University of Palermo, Via Archirafi 34, 90123 Palermo, Italy

E-mail address: dipiazza@math.unipa.it 